\title{
Effect of Shadowing on Outage Probability in Fluid Cellular Radio Networks
}

\author{
Jean-Marc Kelif \\ France Telecom R\&D \\ Issy-Les-Moulineaux, France \\ jeanmarc.kelif@orange-ftgroup.com
}

\author{
Marceau Coupechoux \\ ENST-CNRS LTCI \\ 46, rue Barrault, Paris, France \\ coupecho@enst.fr
}

\author{
Philippe Godlewski \\ ENST-CNRS LTCI \\ 46, rue Barrault, Paris, France \\ godlewsk@enst.fr
}

\begin{abstract}
We propose an adaptive model for the study of cellular networks called the fluid model, useful to each specific network environment characterized by the radio propagation (distance path-loss and shadowing) and by the network configuration. The key idea of the fluid model is to consider the discrete base stations (BS) entities as a continuum of transmitters which are spatially distributed in the network. This allows us to obtain simple analytical expressions of the main characteristics of the network. We focus on the downlink other-cell interference factor, $f$, which is defined here as the ratio of outer cell received power to the inner cell received power. Taking into account the shadowing, $f$ is expressed as a lognormal random variable. Closed-form formulas of the interference factor's mean $m_{f}$ and standard deviation $\sigma_{f}$ are provided in this paper. From $f$, we are able to derive the global outage probability and the spatial outage probability, which depends on the location of a mobile station (MS) initiating a new call. Comparisons to Monte Carlo simulations are proposed, performed in a traditional hexagonal network. Although this factor has been firstly defined for CDMA networks, the analysis presented hereafter is still valid for other systems using frequency reuse 1, like OFDMA (WiMAX), TDMA (GSM with frequency hopping), or even ad hoc networks.
\end{abstract}

\section{INTRODUCTION}

The estimation of cellular networks capacity is one of the key points before deployment and mainly depends on the characterization of interference. As downlink is often the limited link w.r.t. capacity, we focus on this direction throughout this paper, although the proposed framework can easily be extended to the uplink. In cellular networks, an important parameter for this characterization is the other-cell interference factor $f$ (OCIF). The precise knowledge of the OCIF allows the derivation of outage probabilities, capacity evaluation and then, the definition of Call Admission Control mechanisms.

In this paper, we define OCIF as the ratio of total othercell received power to the total inner-cell received power. Pioneering works on the subject [1] were mainly focusing on the uplink. Working on this link, [5] derived the distribution function of a ratio of path-losses, which is essential for the evaluation of external interference. For that, authors approximate the hexagonal cell with a disk of same area. Based on this result, Liu and Everitt propose in [6] an iterative algorithm for the computation of the OCIF, also on the uplink.

On the downlink, [2] [3] aimed at computing an average OCIF over the cell by numerical integration in hexagonal networks. In [8], Gilhousen et al. provide Monte Carlo simulations and obtain an histogram of $f$. In [7], other-cell interference is given as a function of the distance to the BS thanks to Monte-Carlo simulations. Chan and Hanly [10] precisely approximate the distribution of the other-cell interference. They however provide formulas that are difficult to handle in practice. Considering random networks, Baccelli et al. [9] provide spatial blocking probabilities in random networks by using gaussian approximation. OCIF is nevertheless not their main concern. They were the first ones to consider blocking probabilities that are evaluated on each location. Authors of [17] make the choice of considering only the first ring of interferers. They can then express the first ring interference by an approximated formula. Although simple, this approximation is not validated by simulations.

In contrast to previous works in the field, the modelling key of our approach is to consider the discrete BS entities of a cellular network as a continuum. Recently, the authors of [15] described a network in terms of macroscopic quantities such as the node density. The same idea is used in [16] for ad hoc networks. They however assumed a very high density of nodes in both papers and unlimited networks. We show hereafter that our model is accurate even when the density of $\mathrm{BS}$ is very low and the network size is limited (see section III-B).

This paper extends the framework proposed in [11] and [13] that provides a simple closed-form formula for $f$ on the downlink as a function of the distance to the BS, the path-loss exponent, the distance between two BS, and the network size. We validate here the formulas by Monte Carlo simulations and show that it is possible to get a simple outage probability approximation by integrating $f$ over a circular cell. As $f$ is obtained as a function of the distance to the BS, it is possible to derive a spatial outage probability, which depends on the location of a newly initiated call. We moreover generalize the approach developed in [14] by considering the effect of the shadowing on the outage probability analysis.

We first introduce the interference model and notations. Then we present the fluid model and its validation, considering a pathloss only depending on the distance from the transmitter. And using the fluid model, we derive outage probabilities.

Since in a real network, the power received at any point of the system also depends on the local environment (terrain, 
buildings, trees), the radio link can be modelled by a term which depends on the distance $r$ from the transmitter (the lineof-sight path), and the environment (terrain, buildings, trees). The first term depends on the type of the global environment, urban or country, and may moreover depend on the type of cells: macro or micro. The last term, the shadowing, is generally modelled as a lognormal distributed [20] function. We thus propose a refinement of the fluid model taking into account the shadowing and more generally the effects of each specific environment (urban, country, streets buildings).

We establish the interference factor's analytical expressions, mean value and standard deviation, as a lognormal random variable (RV) according to the Fenton-Wilkinson approximation for a sum of lognormal RV [18]. We show the the environment of the system is characterized by a function $H(\sigma)$, and we establish its analytical expression. That function depends on the topology of the network, the exponential pathloss parameter and the shadowing. Using the fluid model network approach, we express the interference factor's mean value and standard deviation, and analyze the influence of different network's parameters: cell radius, exponential pathloss parameter, distance of the mobile to its serving base station.

\section{INTERFERENCE MODEL AND NOTATIONS}

We consider a CDMA system and we focus on the downlink. BS have omni-directional antennas, so that a BS covers a single cell. If a mobile $u$ is attached to a station $b$ (or serving BS), we write $b=\psi(u)$.

The propagation path gain $g_{b, u}$ designates the inverse of the pathloss $p l$ between station $b$ and mobile $u, g_{b, u}=1 / p l_{b, u}$.

The following power quantities are considered:

- $P_{b, u}$ is the transmitted power from station $b$ towards mobile $u$ (for user's traffic);

- $P_{b}=P_{c c h}+\Sigma_{u} P_{b, u}$ is the total power transmitted by station $b, P_{c c h}$ represents the amount of power used to support broadcast and common control channels.

- $p_{b, u}$ is the power received at mobile $u$ from station $b$; we can write $p_{b, u}=P_{b} g_{b, u}$;

- $S_{b, u}=P_{b, u} g_{b, u}$ is the useful power received at mobile $u$ from station $b$ (for traffic data); since we do not consider SHO, we can write $S_{u}=S_{\psi(u), u}$. [11] [13]

The total amount of power experienced by a mobile station $u$ in a cellular system can be split up into several terms: useful signal $\left(S_{u}\right)$, interference and noise (Noise). It is common to split the system power into two terms: $I_{u}=I_{i n t, u}+I_{e x t, u}$, where $I_{\text {int }, u}$ is the internal (or own-cell) received power and $I_{e x t, u}$ is the external (or other-cell) interference. Notice that we made the choice of including the useful signal $S_{u}$ in $I_{i n t, u}$, and, as a consequence, it has to be distinguished from the commonly considered own-cell interference.

With the above notations, we define the interference factor in $u$, as the ratio of total power received from other BS to the total power received from the serving BS $\psi(u): f_{u}=$ $I_{e x t, u} / I_{i n t, u}$. The quantities $f_{u}, I_{e x t, u}$, and $I_{i n t, u}$ are location dependent and can thus be defined in any location $x$ as long as the serving BS is known.
In downlink, orthogonality between physical channels may be approached by Hadamard multiplexing if the delay spread is much smaller than the chip duration $T_{c}$. As a consequence, a coefficient $\alpha$, may be introduced to account for the lack of perfect orthogonality in the own cell.

In this paper, we will use the signal to interference plus noise ratio (SINR) as the criteria of radio quality: $\gamma_{u}^{*}$ is the SINR target for the service requested by MS $u$. This figure is a priori different from the SINR evaluated at mobile station $u$. However, we assume perfect power control, so $S I N R=\gamma_{u}^{*}$ for all users. With the introduced notations, the SINR experimented by $u$ can thus be derivated (see e.g. [4]):

$$
\gamma_{u}^{*}=\frac{S_{u}}{\alpha\left(I_{i n t, u}-S_{u}\right)+I_{e x t, u}+\text { Noise }}
$$

From this relation, we can express $S_{u}$ as:

$$
S_{u}=\frac{\gamma_{u}^{*}}{1+\alpha \gamma_{u}^{*}} I_{i n t, u}\left(\alpha+I_{e x t, u} / I_{\text {int }, u}+\text { Noise } / I_{\text {int }, u}\right)
$$

and the transmitted power for MS $u, P_{b, u}=S_{u} / g_{b, u}$, using relations $I_{i n t, u}=P_{b} g_{b, u}$ and $f=I_{e x t} / I_{\text {int }}$ as:

$$
P_{b, u}=\frac{\gamma_{u}^{*}}{1+\alpha \gamma_{u}^{*}}\left(\alpha P_{b}+f_{u} P_{b}+\text { Noise } / g_{b, u}\right) .
$$

From this relation, the output power of BS $b$ can be computed as follows:

$$
P_{b}=P_{c c h}+\sum_{u} P_{b, u},
$$

and so, according to Eq.3,

$$
P_{b}=\frac{P_{c c h}+\sum_{u} \frac{\gamma_{u}^{*}}{1+\alpha \gamma_{u}^{*}} \frac{\text { Noise }}{g_{b, u}}}{1-\sum_{u} \frac{\gamma_{u}^{*}}{1+\alpha \gamma_{u}^{*}}\left(\alpha+f_{u}\right)} .
$$

\section{FLUID MODEL}

In this section, we first present the model, derive the closedform formula for $f$, and validate it through Monte-Carlo simulations in a hexagonal network.

\section{A. OCIF formula}

The key modelling step of the model we propose consists in replacing a given fixed finite number of BS by an equivalent continuum of transmitters which are spatially distributed in the network. This means that the transmitting power is now considered as a continuum field all over the network. In this context, the network is characterised by a MS density $\rho_{M S}$ and a base station density $\rho_{B S}$ [11]. We assume that MS and BS are uniformly distributed in the network, so that $\rho_{M S}$ and $\rho_{B S}$ are constant. As the network is homogeneous, all base stations have the same output power $P_{b}$.

We focus on a given cell and consider a round shaped network around this centre cell with radius $R_{n w}$. The half distance between two base stations is $R_{c}$ (see Figure 1).

For the assumed omni-directional BS network, we use a propagation model, where the path gain, $g_{b, u}$, only depends on the distance $r$ between the BS $b$ and the MS $u$. The power, $p_{b, u}$, received by a mobile at distance $r_{u}$ can thus be written 


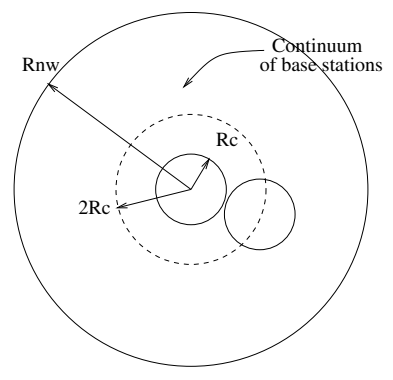

Fig. 1. Network and cell of interest in the fluid model; the distance between two $\mathrm{BS}$ is $2 R_{c}$ and the network is made of a continuum of base stations.

$p_{b, u}=P_{b} K r_{u}^{-\eta}$, where $K$ is a constant and $\eta>2$ is the path-loss exponent.

Let's consider a mobile $u$ at a distance $r_{u}$ from its serving BS $b=\psi(u)$. Each elementary surface $z d z d \theta$ at a distance $z$ from $u$ contains $\rho_{B S} z d z d \theta$ base stations which contribute to $I_{\text {ext,u}}$. Their contribution to the external interference is $\rho_{B S} z d z d \theta P_{b} K z^{-\eta}$. We approximate the integration surface by a ring with centre $u$, inner radius $2 R_{c}-r_{u}$, and outer radius $R_{n w}-r_{u}$ (see Figure 2 ).

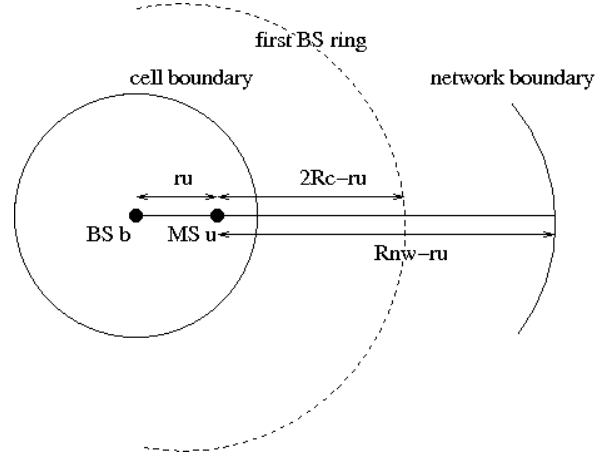

Fig. 2. Integration limits for external interference computation.

$$
\begin{gathered}
I_{e x t, u}=\int_{0}^{2 \pi} \int_{2 R_{c}-r_{u}}^{R_{n w}-r_{u}} \rho_{B S} P_{b} K z^{-\eta} z d z d \theta \\
=\frac{2 \pi \rho_{B S} P_{b} K}{\eta-2}\left[\left(2 R_{c}-r_{u}\right)^{2-\eta}-\left(R_{n w}-r_{u}\right)^{2-\eta}\right] .
\end{gathered}
$$

Moreover, MS $u$ receives internal power from $b$, which is at distance $r_{u}: I_{i n t, u}=P_{b} K r_{u}^{-\eta}$. So, the interference factor $f_{u}=I_{e x t, u} / I_{\text {int }, u}$ can be expressed by:

$$
f_{u}=\frac{2 \pi \rho_{B S} r_{u}^{\eta}}{\eta-2}\left[\left(2 R_{c}-r_{u}\right)^{2-\eta}-\left(R_{n w}-r_{u}\right)^{2-\eta}\right] .
$$

Note that $f_{u}$ does not depend on the BS output power. This is due to the fact that we assumed an homogeneous network and so all base stations emit the same power. In our model, $f$ only depends on the distance $r$ to the BS and can be defined in each location, so that we can write $f$ as a function of $r$ and $\eta, f(r, \eta)$. Thus, if the network is large, i.e. $R_{n w}$ is big in front of $R_{c}, f_{u}$ can be further approximated by:

$$
f(r, \eta)=\frac{2 \pi \rho_{B S} r^{\eta}}{\eta-2}\left(2 R_{c}-r\right)^{2-\eta} .
$$

This closed-form formula will allow us to fastly compute performance parameters of a CDMA network. Moreover, we notice that the fluid analysis of $I_{\text {ext }}$ can be led for different types of cellular networks like OFDMA. However, before going ahead, we need to validate the different approximations we made in this model.

\section{B. Validation of the Fluid Model}

In this section, we aim at validating the fluid model presented above. In this perspective, we will compare the figures obtained with Eq.7 with those obtained numerically by simulations. The simulator assumes an homogeneous hexagonal network made of several rings around a centre cell. Figure 3 shows an example of such a network with the main parameters involved in the study.

The fluid model and the traditional hexagonal model are two simplifications of the reality. None is a priori better than the other but the latter is widely used, especially for dimensioning purposes. That is the reason why a comparison is useful.

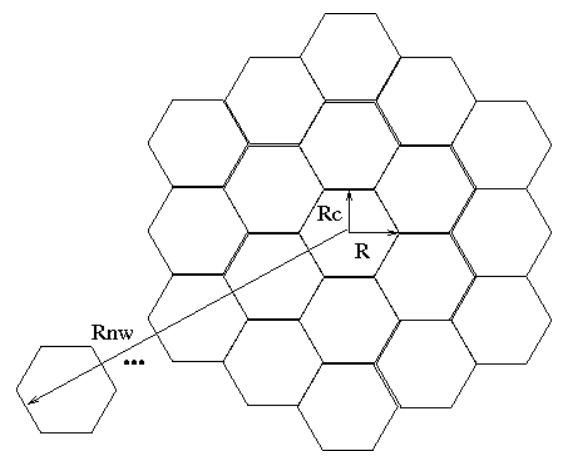

Fig. 3. Hexagonal network and main parameters of the study.

The validation is done numerically by computing $f$ in each point of the cell and averaging the values at a given distance from the BS. This computation can be done independently of the number of MS in the cell and of the BS output power. Factor $f$ indeed depends only on the path-losses to the BS of the network.

Figure 4 shows the simulated interference factor as a function of the distance to the base station. Simulation parameters are the following: $R=1 \mathrm{Km}, \alpha=0.7, \eta$ between 2.7 and $4, \rho_{B S}=\left(3 \sqrt{3} R^{2} / 2\right)^{-1}$, the number of rings is 15 , and the number of snapshots is 1000 . Eq.7 is also plotted for comparison. In all cases, the fluid model matches very well the simulations on an hexagonal network for various figures of the path-loss exponent. It allows calculating the influence of a mobile, whatever its position in a cell.

Note that the considered network size can be finite and chosen to characterize each specific local network's environment. Figure 5 shows the influence of the network size. This model allows thus to develop analyses, adapted to each zone, taking into account each specific considered zone's parameters. 


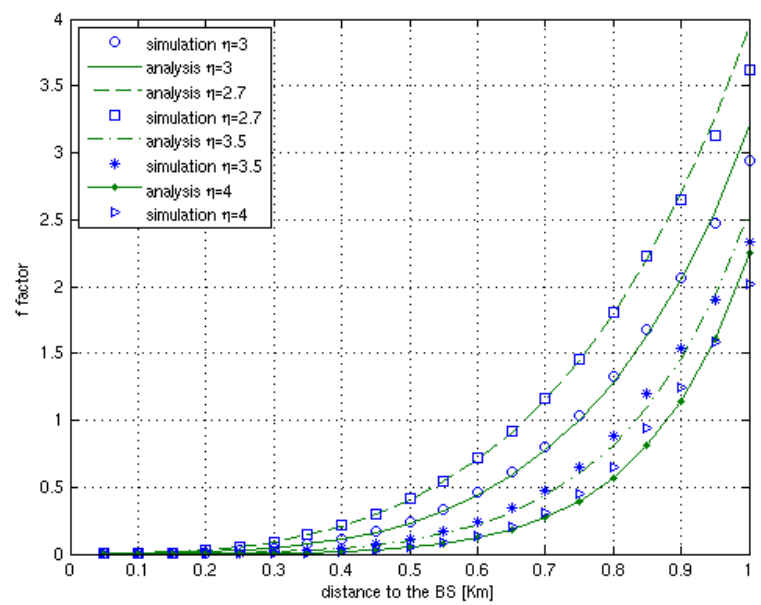

Fig. 4. Interference factor vs. distance to the BS; comparison of the fluid model with simulations on an hexagonal network with $\eta=2.7,3,3.5$, and 4.

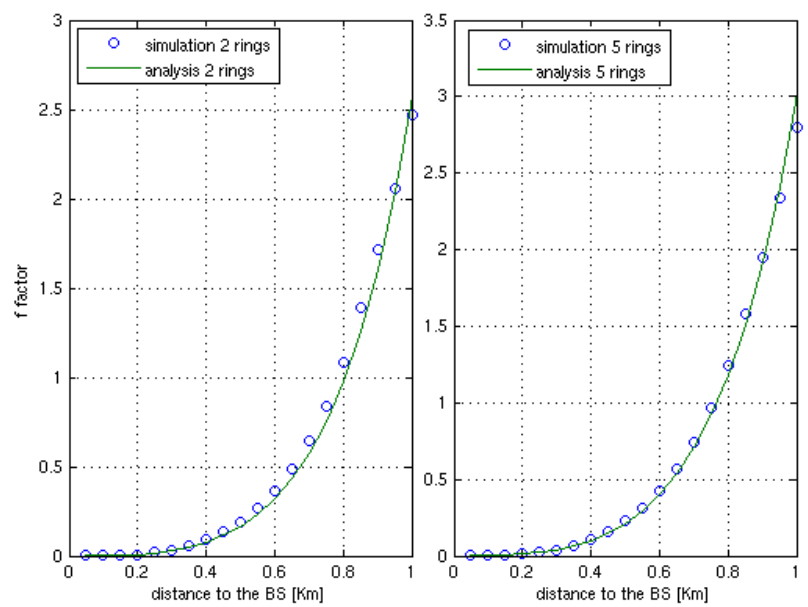

Fig. 5. Interference factor vs. distance to the BS; comparison of the fluid model with simulations on a two ring (left) and a five ring (right) hexagonal network $(\eta=3)$.

We notice moreover that our model can be used even for great distances between the base stations: We validated the model considering a distance of $2 \mathrm{Km}$ between the BS. We conclude that our approach is accurate even for a very low base station's density.

\section{OCIF formula for hexagonal networks}

Two frameworks for the study of cellular networks are considered in this paper: the traditional hexagonal model and the fluid model. While the former is widely used, the latter is very simple and allows the derivation of an analytical formula for $f$. The last section has shown that both models leads to comparable results for the OCIF as a function of the distance to the BS. If we want to go further in the comparison of both models, in particular with the computation of outage probabilities, we need however to be more accurate.

Such calculations require indeed the use of the Gaussian error function $Q$, which is very sensitive to its arguments. This point is rarely raised in litterature: analysis and Monte Carlo simulations can lead to quite different outage probabilities even if analytical average and variance of the underlying Gaussian distribution are very close to simulated figures.

In this perspective, this section provides an alternative formula for $f$ that better matches the simulated figures in an hexagonal network. Note that this result is not needed if network designers use the new framework proposed in this paper. An accurate fitting of analytical and simulated curves shows that $f$ should simply be multiplied by an affine function of $\eta$ to match with Monte Carlo simulations in an hexagonal network. Eq.9 can then be re-written as follows:

$$
f_{\text {hexa }}(r, \eta)=\left(1+A_{\text {hexa }}(\eta)\right) \frac{2 \pi \rho_{B S} r^{\eta}}{\eta-2}\left(2 R_{c}-r\right)^{2-\eta},
$$

where $A_{\text {hexa }}(\eta)=0.15 \eta+0.68$ is a corrective term obtained by least-square fitting.

\section{OUTAGE PROBABILITIES}

The closed-form formula of $f$ allows to compute the global outage probability and the spatial outage probability. Since we provide closed-form formulas for the mean and standard deviation of $f$ over a cell, we use the gaussian approximation.

\section{A. Global outage probability}

For a given number of MS per cell, $n$, outage probability, $P_{\text {out }}^{(n)}$, is the proportion of configurations, for which the needed BS output power exceeds the maximum output power: $P_{b}>$ $P_{\max }$. If noise is neglected and if we assume a single service network $\left(\gamma_{u}^{*}=\gamma^{*}\right.$ for all $\left.u\right)$, we deduce from Eq.5 [12]:

$$
P_{\text {out }}^{(n)}=\operatorname{Pr}\left[\sum_{u=0}^{n-1}\left(\alpha+f\left(r_{u}, \eta\right)\right)>\frac{1-\varphi}{\beta}\right],
$$

where $\varphi=P_{c c h} / P_{\max }$ and $\beta=\gamma^{*} /\left(1+\alpha \gamma^{*}\right)$.

\section{B. Spatial Outage Probability}

For a given number $n$ of MS per cell, a spatial outage probability can also be defined. In this case, it is assumed that $n$ MS have already been accepted by the system, i.e. the output power needed to serve them does not exceed the maximum allowed power. The spatial outage probability at location $r_{u}$ is the probability that maximum power is exceeded if a new MS is accepted in $r_{u}$.

As previously in this paper, we make the approximation that the spatial outage, $P_{\text {sout }}^{(n)}\left(r_{u}\right)$, only depends on the distance to the BS and thus, can be written:

$$
\begin{aligned}
P_{\text {sout }}^{(n)}\left(r_{u}\right)=\operatorname{Pr} & {\left[\left(\alpha+f\left(r_{u}, \eta\right)\right)+\sum_{v=0}^{n-1}\left(\alpha+f\left(r_{v}\right)\right)>\frac{1-\varphi}{\beta} \mid\right.} \\
& \left.\sum_{v=0}^{n-1}\left(\alpha+f\left(r_{v}\right)\right) \leq \frac{1-\varphi}{\beta}\right]
\end{aligned}
$$




$$
=\frac{\operatorname{Pr}\left[\frac{1-\varphi}{\beta}-\left(\alpha+f\left(r_{u}, \eta\right)\right)<\sum_{v=0}^{n-1}\left(\alpha+f\left(r_{v}\right)\right) \leq \frac{1-\varphi}{\beta}\right]}{\operatorname{Pr}\left[\sum_{v=0}^{n-1}\left(\alpha+f\left(r_{v}\right)\right) \leq \frac{1-\varphi}{\beta}\right]}
$$

\section{Gaussian Approximation}

In order to compute these probabilities, we rely on the Central Limit theorem and use a Gaussian approximation. As a consequence, we need to compute the spatial mean and standard deviation of $f\left(r_{u}, \eta\right)$. The area of a cell is $1 / \rho_{B S}=\pi R_{e}^{2}$ with $R_{e}=R_{c} \sqrt{2 \sqrt{3} / \pi}$. So, we integrate $f(r, \eta)$ on a disk of radius $R_{e}$.

As MS are uniformly distributed over the equivalent disk, the probability density function (pdf) of $r$ is: $p_{r}(t)=\frac{2 t}{R_{e}^{2}}$. Let $\mu_{f}$ and $\sigma_{f}$ be respectively the mean and standard deviation of $f(r, \eta)$, when $r$ is uniformly distributed over the disk of radius $R_{e}$.

$$
\begin{aligned}
\mu_{f}= & \frac{2 \pi \rho_{B S}}{\eta-2} \int_{0}^{R_{e}} t^{\eta}\left(2 R_{c}-t\right)^{2-\eta} \frac{2 t}{R_{e}^{2}} d t \\
= & \frac{2^{4-\eta} \pi \rho_{B S} R_{c}^{2}}{\eta-2}\left(\frac{R_{e}}{R_{c}}\right)^{\eta} \int_{0}^{1} x^{\eta+1}\left(1-\frac{R_{e} x}{2 R_{c}}\right)^{2-\eta} d x \\
= & \frac{2^{4-\eta} \pi \rho_{B S} R_{c}^{2}}{\eta^{2}-4}\left(\frac{R_{e}}{R_{c}}\right)^{\eta} \times \\
& { }_{2} F_{1}\left(\eta-2, \eta+2, \eta+3, R_{e} / 2 R_{c}\right),
\end{aligned}
$$

where ${ }_{2} F_{1}(a, b, c, z)$ is the hypergeometric function, whose integral form is given by:

$$
{ }_{2} F_{1}(a, b, c, z)=\frac{\Gamma(c)}{\Gamma(b) \Gamma(c-b)} \int_{0}^{1} \frac{t^{b-1}(1-t)^{c-b-1}}{(1-t z)^{a}} d t,
$$

and $\Gamma$ is the gamma function.

Note that for $\eta=3$, we have the simple closed formula:

$$
\mu_{f}=-2 \pi \rho_{B S} R_{c}^{2}\left(\frac{\ln (1-\nu / 2)}{\nu^{2}}+\frac{16}{\nu}+4+\frac{4 \nu}{3}+\frac{\nu^{2}}{2}\right),
$$

where $\nu=R_{e} / R_{c}$. In the same way, the variance of $f(r, \eta)$ is given by:

$$
\begin{aligned}
\sigma_{f}^{2}= & E\left[f^{2}\right]-\mu_{f}^{2} \\
E\left[f^{2}\right]= & \frac{2^{4-2 \eta}\left(2 \pi \rho_{B S} R_{c}^{2}\right)^{2}}{(\eta+1)(\eta-2)^{2}}\left(\frac{R_{e}}{R_{c}}\right)^{2 \eta} \times \\
& { }_{2} F_{1}\left(2 \eta-4,2 \eta+2,2 \eta+3, \frac{R_{e}}{2 R_{c}} . .\right.
\end{aligned}
$$

As a conclusion of this section, the outage probability can be approximated by:

$$
P_{\text {out }}^{(n)}=Q\left(\frac{\frac{1-\varphi}{\beta}-n \mu_{f}-n \alpha}{\sqrt{n} \sigma_{f}}\right),
$$

where $Q$ is the error function. And the spatial outage probability can be approximated by:

$$
P_{\text {sout }}^{(n)}\left(r_{u}\right)=
$$

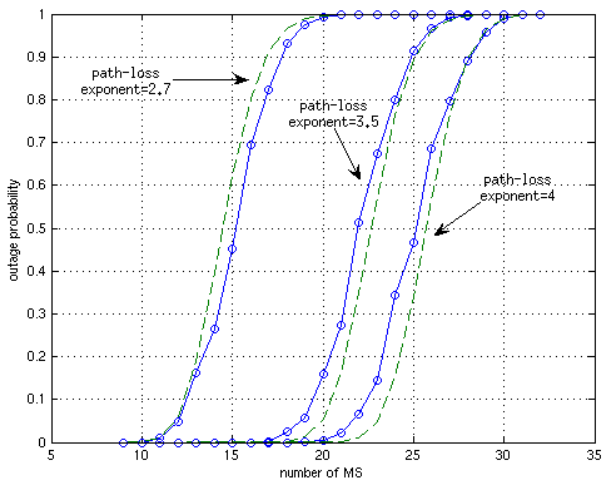

Fig. 6. Global outage probability as a function of the number of MS per cell and for path-loss exponents $\eta=2.7,3.5$ and 4 , simulation (solid lines) and analysis (dotted lines).

$$
\frac{Q\left(\frac{\frac{1-\varphi}{\beta}-n \mu_{f}-(n+1) \alpha-f\left(r_{u}, \eta\right)}{\sqrt{n} \sigma_{f}}\right)-Q\left(\frac{\frac{1-\varphi}{\beta}-n \mu_{f}-n \alpha}{\sqrt{n} \sigma_{f}}\right)}{1-Q\left(\frac{\frac{1-\varphi}{\beta}-n \mu_{f}-n \alpha}{\sqrt{n} \sigma_{f}}\right)},
$$

where $f\left(r_{u}, \eta\right)$ is given by Eq.8. Note that for an accurate fitting of the analytical formulas presented in this section to the Monte Carlo simulations performed in an hexagonal network, $\mu_{f}$ should be multiplied by $\left(1+A_{\text {hexa }}(\eta)\right), \sigma_{f}$ by $\left(1+A_{\text {hexa }}(\eta)\right)^{2}$ and Eq.8 replaced by Eq.9.

\section{Results}

Figures 6 and 8 show the kind of results we are able to obtain instantaneously thanks to the simple formulas derived in this paper for voice service $\left(\gamma_{u}^{*}=-16 \mathrm{~dB}\right)$. Analytical formulas are compared to Monte Carlo simulations in an hexagonal cellular network. As a matter of fact, Eq.9 is used. Figure 6 shows the global outage probabilities as a function of the number of MS per cell for various values of the pathloss exponent $\eta$. It allows us to easily find the capacity of the network at any given maximum percentage of outage. For example, the outage probability when there are 12 users per cell is about $10 \%$ with $\eta=3.5$. Figure 7 shows, as an example, the capacity with $2 \%$ outage as a function of $\eta$.

Figure 8 shows the spatial outage probability as a function of the distance to the BS for $\eta=3$ and for various number of MS per cell. Given that there are already $n$, these curves give the probability that a new user, initiating a new call at a given distance, implies an outage. As an example, a new user in a cell with already 16 on-going calls, will cause outage with probability 0.17 at $900 \mathrm{~m}$ from the BS and with probability 0.05 at $650 \mathrm{~m}$ from the BS.

With this result, an operator would be able to admit or reject new connexions according to the location of the entering MS. Thus, this allows a finer admission control than with the global outage probability.

Until now, the radio link model only expressed that the power received at any point of the system depends on its distance from the transmitter (the line-of-sight path). We show 


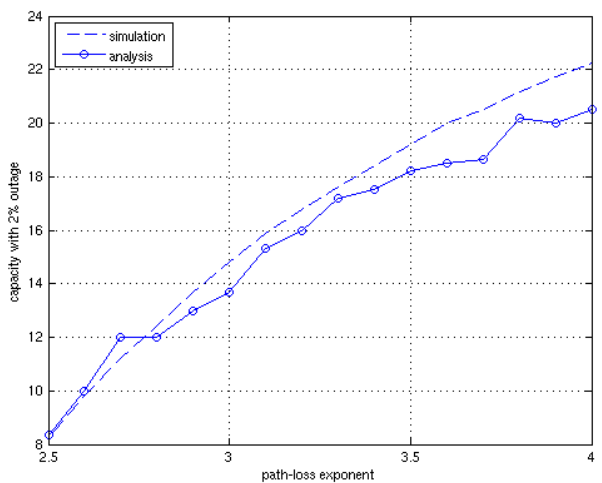

Fig. 7. Capacity with $2 \%$ outage as a function of the path-loss exponent $\eta$, simulations (solid lines) and analysis (dotted lines) are compared.

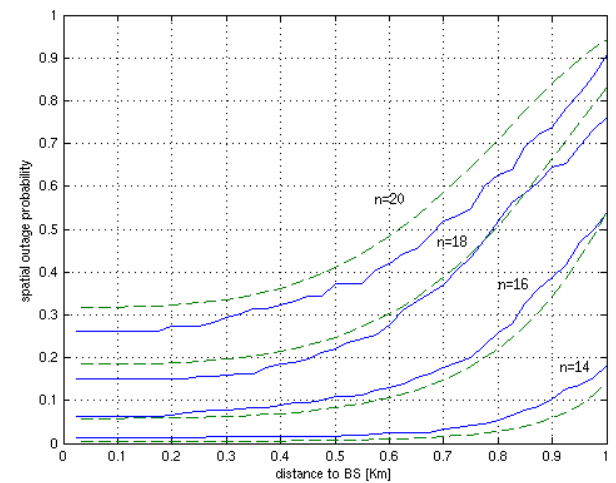

Fig. 8. Spatial outage probability as a function of the distance to the BS for various number of users per cell and for $\eta=3$.

that the shadowing has a limited influence, and we express the interference factor bounds, minimum and maximum.

\section{SHAdOWING INFLUENCE}

\section{A. Propagation}

Considering the power $P_{j}$ transmitted by the $\mathrm{BS} j$, and $r A$ the pathloss including the shadowing effect, the power $p_{j, u}$ received by a mobile $u$ belonging to $j$ can be written:

$$
p_{j, u}=P_{j} K r_{u}^{-\eta} A
$$

$A=10^{\frac{\xi}{10}}$ represents the shadowing effect. The term A characterizes the random variations of the received power around a mean value. $\xi$ is a Normal distributed random variable $R V$, with mean 0 and standard deviation comprised between 0 and $10 \mathrm{~dB}$. The term $P_{j} K r_{u}^{-\eta}$ represents the mean value of the received signal at the distance $r$ from the transmitter $\left(B S_{j}\right)$. The probability density function (PDF) of this slowly varying received signal power is given by

$$
p(s)=\frac{1}{a \sigma s \sqrt{\pi}} \exp -\left(\frac{\ln (s)-m}{\sqrt{2} a \sigma}\right)^{2}
$$

where and
- $a=\frac{\ln 10}{10}$

- $m=\frac{1}{a} \ln \left(K P_{j} r^{-\eta}\right)$ is the (logarithmic) received mean power expressed in decibels $(\mathrm{dB})$, which is related to the path loss and

- $\sigma$ is the (logarithmic) standard deviation of the mean received signal due to the shadowing in decibels.

Remark

We consider a model proposed in [3]. The Rayleigh fading, not considered in this model, may be indistinguishable from shadowing if the fading is sufficiently slow [3], as for example if the mobile travels through a region of deep fades at a very slow speed.

\section{B. Interference power}

Since the interference factor is defined as $f_{u}=$ $I_{\text {ext }, u} / I_{\text {int }, u}$, we first need to calculate the other cell interference power. The total interference power due to all the BS of the network (except the serving one) $I_{\text {ext,u }}=$ $\sum_{i \neq b}^{B} P_{j} g_{j, u}$ is the sum of $N_{B S}$ lognormal $R V$. No exact expression for the PDF of the sum of lognormal distributed RV's is known. It is however accepted that such a sum can be approximated by another lognormal distribution [20]. Among the methods developed to find the mean and variance of that last one, the Schwartz-Yeh approximation [19] is based on a recursive approach. Some descriptions and comparisons of these methods are available in [20]. We choose the FentonWilkinson one [18] for its relative simplicity: the logarithmic mean and the logarithmic variance of a sum of lognormal RV can be found by matching the first and second-order moments. We aim to calculate the interference factor as a lognormal RV (mean and standard deviation). We first calculate the mean and the variance of a sum of lognormal RV, according to the FW method. We afterwards apply the result to the sum of $N_{B S}$ lognormal identically distributed $R V$.

\section{Sum of lognormal $R V$}

Let $X$ be a lognormal $R V$. We can write $\ln X \propto$ $N\left(a m, a^{2} \sigma^{2}\right)$. Let $a Y=\ln X$ we write $a Y \propto N\left(a m, a^{2} \sigma^{2}\right)$. The mean $M$ and the variance $S^{2}$ of a lognormal $R V$, based on a FW approximation [18] [21], are expressed as $M=$ $\exp \left(a m+a^{2} \sigma^{2} / 2\right)$ and $S^{2}=\exp \left(2 a m+a^{2} \sigma^{2}\right) \exp \left(a^{2} \sigma^{2}-1\right)$. So we can write $a m=\ln M-a^{2} \sigma^{2} / 2$ and $a^{2} \sigma^{2}=\ln \left(\frac{S^{2}}{M^{2}}+1\right)$. The sum of lognormal $R V \quad X_{j}\left(M_{j}, S_{j}^{2}\right)$ is written as a lognormal $R V X\left(M, S^{2}\right)$ where $M=M_{j}$ and $S^{2}=S_{j}^{2}$. We can write

$$
a m=\ln \left(\sum_{j} \exp \left(a m_{j}+\frac{a^{2} \sigma_{j}^{2}}{2}\right)\right)-\frac{a^{2} \sigma^{2}}{2}
$$

and

$$
a^{2} \sigma^{2}=\ln \frac{\sum_{j} \exp \left(2 a m_{j}+a^{2} \sigma_{j}^{2}\right)\left(\exp \left(a^{2} \sigma_{j}^{2}\right)-1\right)}{\sum_{j} \exp \left(a m_{j}+a^{2} \sigma_{j}^{2}\right)}
$$




\section{Interference factor}

Our aim is to calculate the interference factor for any mobile at the distance $r_{b}$ from its serving the $B S_{b}$. We can drop the index $\mathrm{b}: r_{b}=r$. Let us consider a network constituted by cells uniformly distributed and a uniform traffic: Each $B S_{j}$ transmits a power $P_{j}$. The power received by a mobile is characterized by a lognormal distribution $X_{j}$ as $\ln X_{j} \propto N\left(a m_{j}, a^{2} \sigma_{j}^{2}\right)$ and we can write $a m_{j}=\ln \left(P_{j} r_{j}\right)$, where $r_{j}$ stands for the distance between the mobile and the $B S_{j}$ of the network. We consider that all the standard deviations are identical $\sigma_{j}=\sigma$ : the total power received by a mobile is a lognormal $R V \mathrm{X}$ characterized by its mean and variance. Expressing the mean interference power received by a mobile, due to all the other base stations of the network (annex 1) and since the ratio of two lognormal $R V$ 's is also expressed as a lognormal $R V$, the interference factor is also lognormally distributed with the following mean $m_{f}$ and logarithmic variance $\sigma_{f}$. Assuming that all the base stations have the same transmitting power $P_{b}=P_{j}=P$ (uniform traffic), we introduce

$$
\begin{gathered}
G(\eta)=\frac{\sum_{j} r_{j}^{-2 \eta}}{\left(\sum_{j} r_{j}^{-\eta}\right)^{2}} \\
f(\eta)=\frac{\sum_{j} r_{j}^{-\eta}}{r^{-\eta}} \\
H(\sigma)=\exp \left(a^{2} \sigma^{2} / 2\right)\left(G(\eta)\left(\exp \left(a^{2} \sigma^{2}\right)-1\right)+1\right)^{\frac{-1}{2}}
\end{gathered}
$$

We can express (see annex 2):

$$
m_{f}=f(\eta) H(\sigma)
$$

The standard deviation is given by

$$
a^{2} \sigma_{f}^{2}=2\left(a^{2} \sigma^{2}-\ln H(\sigma)\right)
$$

We can deduce the interference factor's limits

$$
f(\eta) \leq m_{f} \leq \frac{f(\eta)}{G(\eta)^{\frac{1}{2}}}
$$

and

$$
\sigma_{f}^{2} \leq 2 \sigma^{2}
$$

\section{Remark}

We notice that $f(\eta)$ corresponds to the interference factor $f_{u}$ without shadowing. From (21), we can write $G(\eta)<1$ whatever $\eta$.

\section{TOPOLOGICAL ANALYSIS}

\section{A. Topological characterization}

The expression (24) means that the effect of the environment of any mobile of a cell, on the interference factor, is characterized by a function $H(\sigma)$. This last one depends on the shadowing of the received signals coming from the base stations, and a $G$ factor which depends on the position of the mobile and the characteristics of the network as

- the exponential pathloss parameter $\eta$, which can vary with the topography and more generally with the geographical environment as urban or country, micro or macro cells.

- the base stations positions and number.

It can be interpreted as an environmental form factor $\mathbf{G}$ of the network. Its analytical calculation may be complex. Indeed, its expression depends on the positions of the considered mobile and the base stations. We notice that the form factor can be rewritten, generalizing (22), as:

$$
G(\eta)=\frac{f(2 \eta)}{f(\eta)^{2}}
$$

The shadowing effect consists in increasing the mean value and the standard deviation of the interference factor (26 and (27). This increase is however limited (fig. 10 and Table 2). In a realistic network, $\sigma_{j}$ is generally comprised between 6 and $12 \mathrm{~dB}$.

\section{B. Fluid model approach}

In our fluid model approach of the network, using the interference factor expression of $f(r, \eta)(7)$, we focus on the dependency with $\eta$ (dropping r) and denote it $f(\eta)$. Thus, to go further on our analysis, it appears interesting to express $f(\eta)$ and $G(\eta)$ using the fluid model approach. Since the expression of $f$ also depends on the distance between two neighbors BS $2 R_{c}$ and the size of the network $\mathrm{R}$, it enables to explore these network parameters influences.

We notice the fluid model is thus adaptable to each specific network's zone or environment. Using this model, we can express the form factor $\mathbf{G}$ limits, using (28) .

From the expressions (7) and (8) of $f$ we can write, dropping the dependency with the distance $r$ :

$$
f(\eta)=\frac{2 \pi \rho_{b s} r^{\eta}}{\eta-2}\left[\left(2 R_{c}-r\right)^{2-\eta}-\left(R_{n w}-r\right)^{2-\eta}\right]
$$

When the considered zone's radius is great compared to the cell's one, i.e. $R_{n w}>>R_{c}$, since we have $0<r<R_{c}$ we can write:

$$
-\frac{(-\eta+2)^{2}}{16(-\eta+1)} \leq G(\eta) \leq-\frac{(-\eta+2)^{2}}{4(-\eta+1)}
$$

Table I indicates the limits of the form factor $\mathrm{G}$ as a function of $\eta$. They allow to determine the limits of the interference factors parameters $m_{f}$ and $\sigma_{f}$ 


\begin{tabular}{|c|c|c|c|c|c|}
\hline$\eta$ & 3 & 3.5 & 4 & 4.5 & 5 \\
\hline$G_{\min }$ & 0.03 & 0.06 & 0.08 & 0.11 & 0.14 \\
\hline$G_{\max }$ & 0.12 & 0.22 & 0.33 & 0.45 & 0.56 \\
\hline
\end{tabular}

TABLE I

FORM FACTOR G LIMITS

\section{Interference factor distance dependency}

The figure (9) shows the influence of the distance between the mobile and its serving BS, for different standard deviations and $\eta=3$ : the mean value of the interference factor increases when the standard deviation increases. Compared to a case without shadowing, and for a mobile located at the edge of the cell $(1000 \mathrm{~m})$, that increase is about $30 \%$ when $\sigma=4 \mathrm{~dB}$ and reaches about $100 \%$ when $\sigma=12 d B$. We observe that the shadowing seems to "increase" the distances from the BS: with a shadowing $\sigma=12 d B$, a mobile at $800 \mathrm{~m}$ from its serving $\mathrm{BS}$ has the same mean interference factor as a mobile at 1000 $\mathrm{m}$ without shadowing. This effect explains the importance of considering shadowing margins during the planning process.

\section{Interference factor standard deviation dependency}

For low variances, i.e. $a^{2} \sigma^{2} \approx 1$, we can express from (23) and (24)

$m_{f} \approx f(\eta) \exp \left(a^{2} \frac{\sigma^{2}}{2}\right)$ and $a^{2} \sigma_{f}^{2} \rightarrow a^{2} \sigma^{2}$.

And for high variances, i.e. $\exp \left(a^{2} \sigma^{2}\right)>>1$ or $\sigma^{2}>>\frac{1}{a^{2}}$, we can write

$m_{f} \approx \frac{f(\eta)}{G^{\frac{1}{2}}}$ and $a^{2} \sigma_{f}^{2} \approx 2 a^{2} \sigma^{2}+\ln (G)$.

For low standard deviations (less then 4 or $5 \mathrm{~dB}$ ), these expressions show a low dependency of the mean value of $f$ with $\sigma$, and the total standard deviation $\sigma_{f}$ is very close to $\sigma$ (Table II).

These expressions show another interesting result: for high variances (higher than $5 \mathrm{~dB}$ ), the interference factor's mean tends towards a value which does not depend on the variance. Considering the extreme $\mathrm{G}$ values (Table $\mathrm{I}$ ), with $\eta=3$, the figure (10) confirms that the mean value of the interference factor increases with the variance BS, until a standard deviation of about $10 \mathrm{~dB}$. For higher values, the interference factor stays constant: we observe the mean interference factor does no more depend on them. Moreover, the form factor compensates the standard deviation influence: it increases with the distance $\mathrm{r}$, and also with the exponential pathloss parameter $\eta$. It means that a high value of that parameter, characterizing a given type of cells or environment, may compensate the shadowing effects(see also Fig. 10). Considering the range of variations of $\sigma$, for a realistic network $\sigma$ is generally comprised between 6 and $12 \mathrm{~dB}$, Table II shows that the standard deviation of the interference factor is close to the BS ones $\sigma$ (with $\eta=3$ ).

\section{E. Interference factor environmental dependency}

The exponential pathloss parameter $\eta$ can characterize the environment type, urban or country, and the cell dimensions

\begin{tabular}{|c|c|c|c|c|c|c|c|c|c|}
\hline$\sigma$ & 0 & 1 & 2 & 3 & 4 & 5 & 8 & 10 & 12 \\
\hline$\sigma_{f}$ & 0 & 1.1 & 2.2 & 3.3 & 4.5 & 5.8 & 10.1 & 13.1 & 16.1 \\
\hline
\end{tabular}

TABLE II

STANDARD DEVIATION OF THE INTERFERENCE FACTOR VS $\sigma$

(pico, micro, macro). The figure (10) confirms that the influence of the shadowing on the mean interference factor decreases when $\eta$ increases. For $\eta \geq 5$ we observe the shadowing has almost no influence.

\section{F. Interference factor cell radius dependency}

Fig. (12) shows the mean interference factor for a mobile at a given relative position $r / R_{c}$ in the cell from its serving base station. When the cell radius $R_{c}$ increases (i.e. the distance between two BS increases) the influence of the shadowing decreases, and becomes very low for a cell's radius higher than $1700 \mathrm{~m}$.

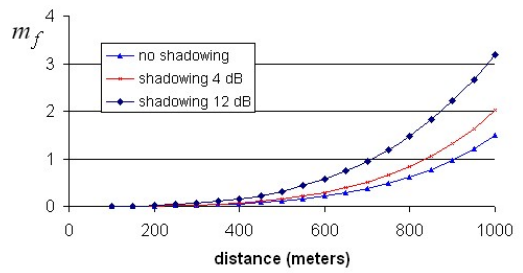

Fig. 9. Influence of the standard deviation on the mean interference factor $m_{f}$ for each distance.

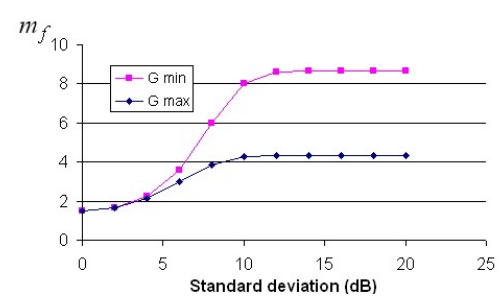

Fig. 10. Mean interference factor $m_{f}$ vs deviation $\sigma($ distance $=1000 \mathrm{~m})$.

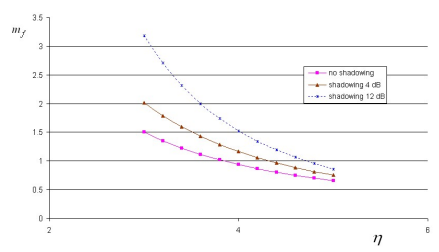

Fig. 11. Mean interference factor vs $\eta$ (edge of the cell).

\section{OUTAGE PROBABILITY: SHADOWING INFLUENCE}

We write the expression (10), considering the fluid model approach and a mobile density $\rho_{M S}$ in the cell. The outage probability is expressed as: 


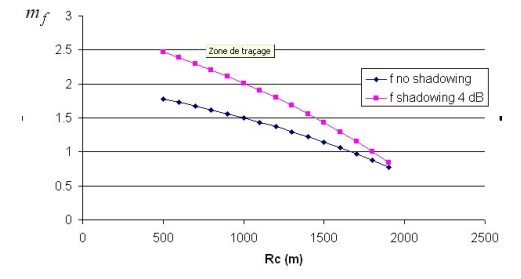

Fig. 12. Mean Interference factor for Identical relative positions (edge of the cell) and $\eta=3$.

$$
P_{\text {out }}=\operatorname{Pr}\left[\int_{0}^{2 \pi} \int_{0}^{R} \rho_{M S}(\alpha+f(r, \eta)) r d r d \theta>\frac{1-\varphi}{\beta}\right],
$$

Let's introduce

$$
C(f)=\int_{0}^{2 \pi} \int_{0}^{R} \rho_{M S}(\alpha+f(r, \eta)) r d r d \theta,
$$

where $f(r, \eta)$ is a lognormal random variable with mean $m_{f}$ and standard deviation $\sigma_{f}$. Considering the PDF $p_{C(f)}$ of $C(f)$ we can write:

$$
P_{\text {out }}=\operatorname{Pr}\left[C(f)>\frac{1-\varphi}{\beta}\right]=\int_{\frac{1-\varphi}{\beta}}^{\infty} p_{C(f)}(x) d x,
$$

A mobile asking for admission in the cell at the distance $r$ from the serving BS, which already manages mobiles with a density $\rho_{M S}$, induces a load $\beta(\alpha+f(r, \eta))$. So we can write

$$
C_{2}(f)=\beta(\alpha+f(r, \eta))+\int_{0}^{2 \pi} \int_{0}^{R} \beta \rho_{M S}(\alpha+f(r, \eta)) r d r d \theta,
$$

The blocking probability of this mobile is thus given by

$$
1-\operatorname{Pr}\left[C_{2}(f) \leq 1-\varphi\right]
$$

Analogue expressions can be derived for the spatial outage probability. Since the interference factor depends on the mobile location and on the environment (expressions 24 and 25) i.e.

- the shadowing,

- the exponential pathloss parameter $\eta$ : It depends on different characteristics and particularly the cell dimensions and the type of environment (urban or country),

- the number and positions of the base stations,

it becomes possible to analyze the outage taking into account the mobile's location and each specific environment. Moreover, in our model, the expression of $f(r, \eta)$ is characterized by the zone (or network) size R. It thus can be adapted and applied to each zone of a network.

\section{CONCLUSION}

We developed an adaptive model of cellular networks, useful to each specific network environment characterized by the radio propagation (distance path-loss and shadowing) and the network configuration.

We first proposed and validated by Monte Carlo simulations a fluid model for the estimation of the OCIF in cellular networks. This approach considers BS as a continuum of transmitters and provides a simple formula for the othercell interference factor as a function of the distance to the BS, the path-loss exponent, the distance between BS and the network size. Simulations show that the obtained closedform formula is a very good approximation, even for the traditional hexagonal network. The simplicity of the result allows a spatial integration of $f$ leading to closed-form formula for the global outage probability and for the spatial outage probability. The proposed framework is not only a powerful tool to study admission control in CDMA networks and design fine algorithms taking into account the distance to the BS. It can also easily be extended to other cellular networks, e.g. to study frequency reuse schemes in OFDMA systems.

Considering the shadowing, we established the OCIF analytical expression, mean $m_{f}$ and standard deviation $\sigma_{f}$, as a lognormal random variable. We expressed them using the fluid model approach, and showed they depend on a function of the shadowing $H(\sigma)$ and a form factor $\mathrm{G}$. This last one depends on the mobile's location, the exponential pathloss parameter, the number and the positions of the base stations. We finally analyzed different environmental parameters influences, and show the form factor, the exponential pathloss parameter and the cell radius may limit the shadowing effects. We finally expressed the outage probability of mobiles, taking into account the shadowing influence.

\section{ANNEX 1: INTERFERENCE POWER}

Each BS transmits a power $P_{j}=P$ so the power received by a mobile is characterized by a lognormal distribution $X_{j}$ as $\ln \left(X_{j}\right) \propto N\left(a m_{j}, a^{2} \sigma_{j}^{2}\right)$ and we can write $m_{j}=\frac{1}{a} \ln \left(P_{j} r_{j}^{-\eta}\right)$ (to simplify the calculation we consider $\mathrm{K}=1$ ). So the total power received by a mobile is a lognormal $R V \mathrm{X}$ characterized by its mean and variance $\ln (X) \propto N\left(a m, a^{2} \sigma_{t}^{2}\right)$ and we can write:

$$
a m=\ln \left(\sum_{j=1, j \neq b}^{B} \exp \left(\ln P_{j}-\eta \ln r_{j}+\frac{a^{2} \sigma_{j}^{2}}{2}\right)\right)-\frac{a^{2} \sigma_{t}^{2}}{2}
$$

We assume identical variance, $\sigma_{j}=\sigma, \forall j$. Since moreover $P_{j}=P$ whatever the base station $\mathrm{j}$ :

$$
a m=\left(\ln P+\frac{a^{2} \sigma^{2}}{2}\right)+\ln \left(\sum_{j=1, j \neq b}^{B} \exp \left(-\eta \ln \left(r_{j}\right)\right)\right)-\frac{a^{2} \sigma_{t}^{2}}{2}
$$


So we can express the mean interference power $\overline{I_{e x t}}$ received by a mobile coming from all the other base stations of the network as:

$$
\ln \left(\overline{I_{e x t}}\right)=\left(\ln P+\frac{a^{2} \sigma^{2}}{2}\right)+\ln \left(\sum_{j=1, j \neq b}^{B} r_{j}^{-\eta}\right)-\frac{a^{2} \sigma_{t}^{2}}{2}
$$

and the variance $a^{2} \sigma_{t}^{2}$ of the sum of interferences is written as

$$
a^{2} \sigma_{t}^{2}=\ln \left(\frac{\sum_{j} \exp \left(2 a m_{j}+a^{2} \sigma^{2}\right)\left(\exp \left(a^{2} \sigma^{2}\right)-1\right)}{\left(\sum_{j} \exp \left(a m_{j}+\frac{a^{2} \sigma^{2}}{2}\right)\right)^{2}}+1\right)
$$

Introducing

$$
G(\eta)=\frac{\sum_{j=1, j \neq b}^{B} r_{j}^{-2 \eta}}{\left(\sum_{j=1, j \neq b}^{B} r_{j}^{-\eta}\right)^{2}}
$$

the mean value of the total interference received by a mobile is given by

$$
\overline{I_{e x t}}=P \sum_{j=1, j \neq b}^{B} r_{j}^{-\eta} \exp \left(\frac{a^{2} \sigma^{2}}{2}\right)\left(\left(\exp \left(a^{2} \sigma^{2}\right)-1\right) G(\eta)+1\right)^{-1 / 2}
$$

and

$$
a^{2} \sigma^{2}=\ln \left(\left(\exp \left(a^{2} \sigma^{2}\right)-1\right) G(\eta)+1\right)+\ln \left(\exp \left(a^{2} \sigma^{2}\right)\right)
$$

\section{ANNEX 2: INTERFERENCE FACTOR}

Since the ratio of two lognormal RV's is also a lognormal $\mathrm{RV}$, the interference factor is also lognormally distributed with the following mean and logarithmic variance:

$$
m_{f}=\frac{\overline{I_{e x t}}}{\overline{I_{i n t}}}
$$

and thus, if we consider that all the base stations have the same transmitting power: $P_{b}=P$, we can write, dropping the index $b$ :

$m_{f}=\frac{\sum_{j} r_{j}^{-\eta}}{r^{-\eta}} \exp \left(\frac{a^{2} \sigma^{2}}{2}\right)\left(\left(\exp \left(a^{2} \sigma^{2}\right)-1\right) G(\eta)+1\right)^{-1 / 2}$

and finally, denoting:

$$
H(\sigma)=\exp \left(\frac{a^{2} \sigma^{2}}{2}\right)\left(\left(\exp \left(a^{2} \sigma^{2}\right)-1\right) G(\eta)+1\right)^{-1 / 2}
$$

we have

$$
m_{f}=f(\eta) \exp \left(\frac{a^{2} \sigma^{2}}{2}\right)\left(\left(\exp \left(a^{2} \sigma^{2}\right)-1\right) G(\eta)+1\right)^{-1 / 2}
$$

In a analogue analysis, the standard deviation is given by $a^{2} \sigma_{f}^{2}=a^{2} \sigma_{f}^{2}+a^{2} \sigma_{j}^{2}$ so we have

$$
a^{2} \sigma_{f}^{2}=2\left(a^{2} \sigma^{2}-\ln (H(\sigma))\right)
$$

\section{REFERENCES}

[1] A. M. Viterbi and A. J. Viterbi, Erlang Capacity of a Power Controlled CDMA System, IEEE J. on Selected Areas in Communications, Vol. 11, No. 6, Aug. 1993.

[2] A. J. Viterbi, A. M. Viterbi, and E. Zehavi, Other-Cell Interference in Cellular Power-Controlled CDMA, IEEE Trans. on Communications, Vol. 42, No. 2/3/4, Freb/Mar/Apr. 1994.

[3] A. J. Viterbi, CDMA - Principles of Spread Spectrum Communication, Addison-Wesley, 1995.

[4] X. Lagrange, Principes et évolutions de 1'UMTS, Hermes, 2005.

[5] J. S. Evans and D. Everitt, Effective Bandwidth-Based Admission Control for Multiservice CDMA Cellular Networks, IEEE Trans. on Vehicular Technology, Vol. 48, No. 1, Jan. 1999.

[6] T. Liu and D. Everitt, Analytical Approximation of Other-Cell Interference in Uplink of CDMA Cellular Systems, Proc. of IEEE VTC Spring, May 2006.

[7] S. E. Elayoubi and T. Chahed, Admission Control in the Downlink of WCDMA/UMTS, Lect. notes comput. sci., Springer, 2005.

[8] K. S. Gilhousen, I. M. Jacobs, R. Padovani, A. J. Viterbi, L. A. Weaver, and C. E. Wheatley, On the Capacity of Cellular CDMA System, IEEE Trans. on Vehicular Technology, Vol. 40, No. 2, May 1991.

[9] F. Baccelli, B. Blaszczyszyn, and M. K. Karray, Blocking Rates in Large CDMA Networks via a Spatial Erlang Formula, Proc. of IEEE INFOCOM, Mar. 2005.

[10] C. C. Chan and Hanly, Calculating the Outage Probabability in CDMA Network with Spatial Poisson Traffic, IEEE Trans. on Vehicular Technology, Vol. 50, No. 1, Jan. 2001.

[11] J.-M. Kelif, Admission Control on Fluid CDMA Networks, Proc. of WiOpt, Apr. 2006.

[12] J.-M. Kelif, Stratégies d'allocations équitables de ressources, Private Communication, 2002.

[13] J-M. Kelif and E. Altman, Downlink Fluid Model of CDMA Networks, Proc. of IEEE VTC Spring, May 2005.

[14] J-M Kelif, Marceau Coupechoux and Philippe Godlewski, Spatial Outage Formula for CDMA Networks, VTC Fall 2007.

[15] S. Toumpis and L. Tassiulas, Packetostatics Deployment of Massively Dense Sensor Networks as an Electrostatics Problem, proc. of INFOCOM, Mar. 2005.

[16] P. Jacquet, Geometry of information propagation in massively dense ad hoc networks, Proc. of ACM MobiHoc, May 2004.

[17] T. Bonald and A. Proutiere Wireless Downlink Data Channels: User Performance and Cell Dimensioning, Proc. of ACM Mobicom 2003.

[18] L.Fenton "The sum of lognormal probability distributions in scatter transmission system", IEEE (IRE) Transactions on Communications, CS8,1960

[19] S. Shwartz and Y.S. Yeh, On the distributions functions and moments of power sums with lognormal components, Bell Syst, Tech J, vol 61, pp 1441 1462, Sept 1982

[20] G.L. Stuber, principles of Mobiles Communications, Norwell , MA. Kluwer, 1996

[21] Fatih Alagz, Approximations on the Aggregate MPEG Video, Traffic and Their Impact on Admission Control, Turk J Elec Engin, Vol 10, N1, 2002

[22] Mohamed-Slim Alouini and Andrea J. Goldsmith, Area Spectral Efficiency of Cellular, Mobile Radio Systems, IEEE Transactions on vehicular technology, Vol. 48, N. 4, 1999 